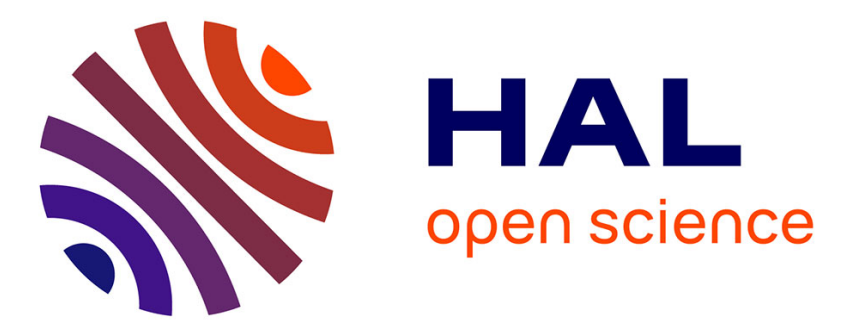

\title{
Rôle éventuel des bilacunes sur l'autodiffusion volumique dans les phases $\beta$ de structure A2
}

\author{
A. Jennane, Ch. Lexcellent, G. Moya, J. Bernardini
}

\section{To cite this version:}

A. Jennane, Ch. Lexcellent, G. Moya, J. Bernardini. Rôle éventuel des bilacunes sur l'autodiffusion volumique dans les phases B de structure A2. Journal de Physique IV Proceedings, 1996, 06 (C2), pp.C2-103-C2-107. 10.1051/jp4:1996214 . jpa-00254192

\section{HAL Id: jpa-00254192 https://hal.science/jpa-00254192}

Submitted on 1 Jan 1996

HAL is a multi-disciplinary open access archive for the deposit and dissemination of scientific research documents, whether they are published or not. The documents may come from teaching and research institutions in France or abroad, or from public or private research centers.
L'archive ouverte pluridisciplinaire HAL, est destinée au dépôt et à la diffusion de documents scientifiques de niveau recherche, publiés ou non, émanant des établissements d'enseignement et de recherche français ou étrangers, des laboratoires publics ou privés. 


\title{
Rôle éventuel des bilacunes sur l'autodiffusion volumique dans les phases $\beta$ de structure A2
}

\author{
A. Jennane*,**, Ch. Lexcellent ***, G. Moya** et J. Bernardini* \\ *Métallurgie, URA 443, Faculté St. Jérôme, 13397 Marseille cedex 20, France \\ ** Physique des Matériaux, EA 882, Faculté St. Jérôme, 13397 Marseille cedex 20, France \\ *** Mécanique Appliquée, URA 004, Faculté des Sciences, 25030 Besançon, France
}

\begin{abstract}
Diffusion of $65 \mathrm{Zn}$ in $\mathrm{Cu}-\mathrm{Zn}$ alloys (48,0at \% $\mathrm{Zn}$ ) in the disordered $\mathrm{A} 2$ phase does not present an Arrhenius behaviour. The diffusion parameters are $D_{0}=0,0046 \mathrm{~cm}^{2} / \mathrm{s}$ and $\mathrm{Q}=82182 \mathrm{~J} /$ Mole between $508-692^{\circ} \mathrm{C}$ and $\mathrm{D}_{0}=0,01 \mathrm{~cm}^{2} / \mathrm{s}$ and $\mathrm{Q}=105557 \mathrm{~J} /$ Mole between $692-826^{\circ} \mathrm{C}$ respectively. Such a behavior might be explained by the presence of divacancies as it exist in the literature experimental evidence of divacancy mechanism of brass dezincification.
\end{abstract}

\section{INTRODUCTION}

En étudiant la diffusion du cuivre et du zinc dans l'alliage $\mathrm{Cu}-\mathrm{Zn}\left(45,6\right.$ at\% $-48,0$ at $\%$ de zinc) entre $840^{\circ} \mathrm{C}$ et $250^{\circ} \mathrm{C}$, Kuper et al. [1] se sont surtout attachés à montrer qu'au dessous de la température de transition ordre-désordre $\mathrm{A} 2 \rightarrow \mathrm{B} 2\left(\mathrm{~T}_{\mathrm{C}}=468^{\circ} \mathrm{C}\right)$, la diffusion des deux éléments était nettement ralentie. Ce phénomène a depuis été retrouvé pour d'autres alliages présentant cette transition et en particulier récemment dans l'alliage $\mathrm{Fe}-\mathrm{Co}[2]$. Il faut noter que dans l'étude présentée par Kuper et al. peu de mesures ont été effectuées dans la phase désordonnée A2 et que ces auteurs obtiennent à partir de quatre valeurs du coefficient de diffusion du $65 \mathrm{Zn}$, mesurées entre 499 et $718^{\circ} \mathrm{C}$, un terme de fréquence $\mathrm{D}_{\mathrm{O}}=0,0035 \mathrm{~cm}^{2} / \mathrm{s}$ et une énergie d'activation $\mathrm{Q}=78584 \mathrm{~J} / \mathrm{mol}$. Ces paramètres sont bien plus faibles que ceux déterminés par Inman et al. [3] entre 636 et $870^{\circ} \mathrm{C}$ au moyen de cinq coefficients de diffusion du $65 \mathrm{Zn}: \mathrm{D}_{\mathrm{o}}=0,024 \mathrm{~cm}^{2} / \mathrm{s} \mathrm{et}$ $\mathrm{Q}=95530 \mathrm{~J} / \mathrm{mol}$. Dans la mesure où ces deux études n'ont pas été effectuées i) sur des alliages présentant la même teneur en zinc, ii) dans le même domaine de température, iii) à de nombreuses températures, il nous a semblé utile de remesurer la diffusion volumique du zinc, surtout dans le domaine désordonné.

\section{TECHNIQUES EXPERIMENTALES}

L'alliage polycristallin cuivre-zinc, à 48,0 at\% de zinc, a été fabriqué par le "Comptoir Lyon-Alemand"avec une taille de grain de 5 à $6 \mathrm{~mm}^{2}$. Les échantillons $\left(1 \mathrm{~cm}^{2}\right.$ de surface et $0,7 \mathrm{~cm}$ d'épaisseur $)$ ont été polis mécaniquement avant de subir un recuit à $850^{\circ} \mathrm{C}$ en ampoules de quartz scellées sous un mélange ArgonHydrogène de pureté $5 \mathrm{~N}$. A près un nouveau polissage des échantillons, ce traitement haute température est suivi d'un traitement de mise en équilibre à la température qui sera celle du traitement de diffusion. Pour éviter autant que possible l'évaporation du zinc, les échantillons encapsulés dans des feuilles de tantale sont en présence d'autres morceaux d'alliage qui servent à établir les pressions d'équilibre. Le radioélément $65 \mathrm{Zn}$, d'activité spécifique égale à $2 \mathrm{mCi} / \mathrm{mg}$, est déposé sur les échantillons par électrolyse. Les échantillons sont ensuite réencapsulés dans une feuille de tantale et remis dans une ampoule de quartz pour subir le recuit de diffusion dans les mêmes conditions que celles relatives au recuit de mise en équilibre. Dans ces conditions, la perte en zinc, estimée par pesée des échantillons après tous les traitements thermiques, a été limitée à $0,1 \%$ environ.

Pour mieux tester le rôle possible de l'évaporation du zinc, quelques expériences ont été effectuées en recouvrant la face active des échantillons par un autre échantillon de même taille avant d'encapsuler l'ensemble de ce "sandwich" dans la feuille de tantale. Les recuits de diffusion ont été réalisés entre $350^{\circ} \mathrm{C}$ et $826^{\circ} \mathrm{C}$ dans des fours pré-régulés et les échantillons ont été trempés en fin de recuit.

Les profils de concentration du radioélément ont été obtenus par la technique de sectionnement en effectuant en alternance des découpages parallèles au front de diffusion et des mesures d'activités au moyen d'un détecteur à scintillation $\mathrm{NaI}$. Les coefficients de diffusion sont déduits des profils de pénétration relatifs à l'évolution de l'activité spécifique $A_{S}$ des tranches découpées en utilisant comme solution de l'équation de Fick la relation: 


$$
A_{s}(X)=\frac{A}{\sqrt{ } \pi D t} \exp \left(\frac{-x^{2}}{4 D t}\right)
$$

où $A$ est l'activité totale du traceur par unité de surface à $x=0$ et $t=0, t$ le temps de diffusion, $D$ le coefficient de diffusion et $x$ la profondeur de pénétration.

\section{RESULTATS EXPERIMENTAUX}

\subsection{Détermination des coefficients de diffusion}

Quelques profils de pénétration expérimentaux sont reportés sur la figure 1. Ils correspondent bien à ceux prévus par la loi théorique.
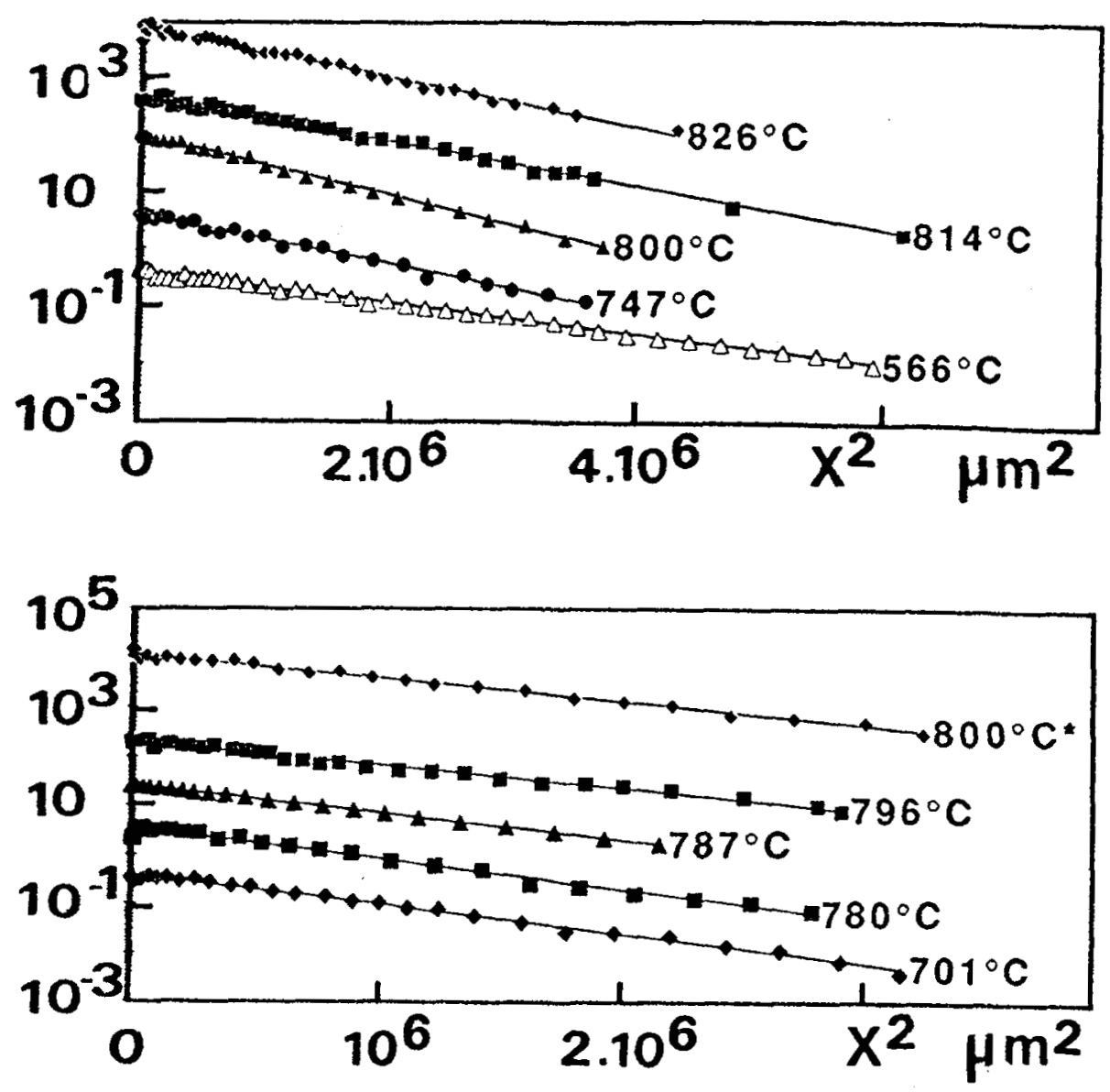

Figure 1: Profils de pénétration du $65 \mathrm{Zn}$ dans l'alliage $\mathrm{Cu}-\mathrm{Zn}(48,0$ at $\%$ en zinc).

L'ensemble des valeurs de $\mathrm{D}$ obtenues à différentes températures est reporté dans le tableau I. On peut noter que les résultats sont reproductibles à $\pm 10 \%\left(508^{\circ} \mathrm{C}\right.$ et $\left.800^{\circ} \mathrm{C}\right)$ et que la présence d'un échantillon de $\mathrm{CuZn}$ sur l'échantillon actif ne modifie par les résultats. 


\begin{tabular}{|c|c|c|c|c|c|}
\hline $\mathrm{T}\left({ }^{\circ} \mathrm{C}\right)$ & $\mathrm{D}\left(\mathrm{cm}^{2} / \mathrm{s}\right)$ & $\sqrt{\mathrm{Dr}}\left(10^{-4} \mathrm{~cm}\right)$ & $\mathrm{T}\left({ }^{\circ} \mathrm{C}\right)$ & $\mathrm{D}\left(\mathrm{cm}^{2} / \mathrm{s}\right)$ & $\sqrt{\mathrm{Dt}}\left(10^{-4} \mathrm{~cm}\right)$ \\
\hline 350 & $4,110^{-11}$ & 65 & 692 & $1,710^{-7}$ & 246 \\
\hline 422 & $8,210^{-10}$ & 71 & 701 & $2,010^{-7}$ & 422 \\
\hline $508^{*}$ & $1,510^{-8}$ & 237 & 747 & $4,310^{-7}$ & 510 \\
\hline 508 & $1,810^{-8}$ & 260 & 780 & $5,910^{-7}$ & 416 \\
\hline 526 & $2,110^{-8}$ & 256 & 787 & $6,610^{-7}$ & 501 \\
\hline 554 & $2,610^{-8}$ & 199 & 796 & $6,110^{-7}$ & 480 \\
\hline 566 & $3,810^{-8}$ & 630 & $800^{*}$ & $7,110^{-7}$ & 477 \\
\hline 600 & $4,710^{-8}$ & 127 & 800 & $6,410^{-7}$ & 460 \\
\hline 634 & $8,710^{-8}$ & 182 & 814 & $8,610^{-7}$ & 530 \\
\hline 650 & $1,210^{-7}$ & 218 & 826 & $9,010^{-7}$ & 510 \\
\hline 676 & $1,410^{-7}$ & 220 & & & \\
\hline
\end{tabular}

* Echantillons recouverts d'un morceau de $\mathrm{Cu}-\mathrm{Zn}$

Tableau. 1 : Coefficients de diffusion du zinc dans $\mathrm{CuZn}(48,5 \% \mathrm{Zn})$.

\section{2 Détermination des paramètres de diffusion}

Sur la figure 2 , l'ensemble des résultats expérimentaux limité à $\mathrm{T}>\mathrm{T}_{\mathrm{C}}$ a été reporté en tenant compte d'une erreur de $\pm 10 \%$ sur la valeur des coefficients de diffusion.

Les erreurs commises sur D proviennent de la mesure du temps de traitement de diffusion (négligeable), de la mesure de la surface ( $2 \%$ ), de la masse de l'échantillon (deux centièmes de milligramme) et du comptage du radioélément (2\%). Ces erreurs sont facilement calculables. Par contre, l'erreur qui pourrait être due au découpage de l'échantillon dans une direction légèrement différente de celle relative au front de diffusion est difficilement chiffrable. Nous avons donc estimé l'erreur sur le coefficient de diffusion a \pm $10 \%$, ce qui correspond à la reproductibilité que nous avons observée expérimentalement.
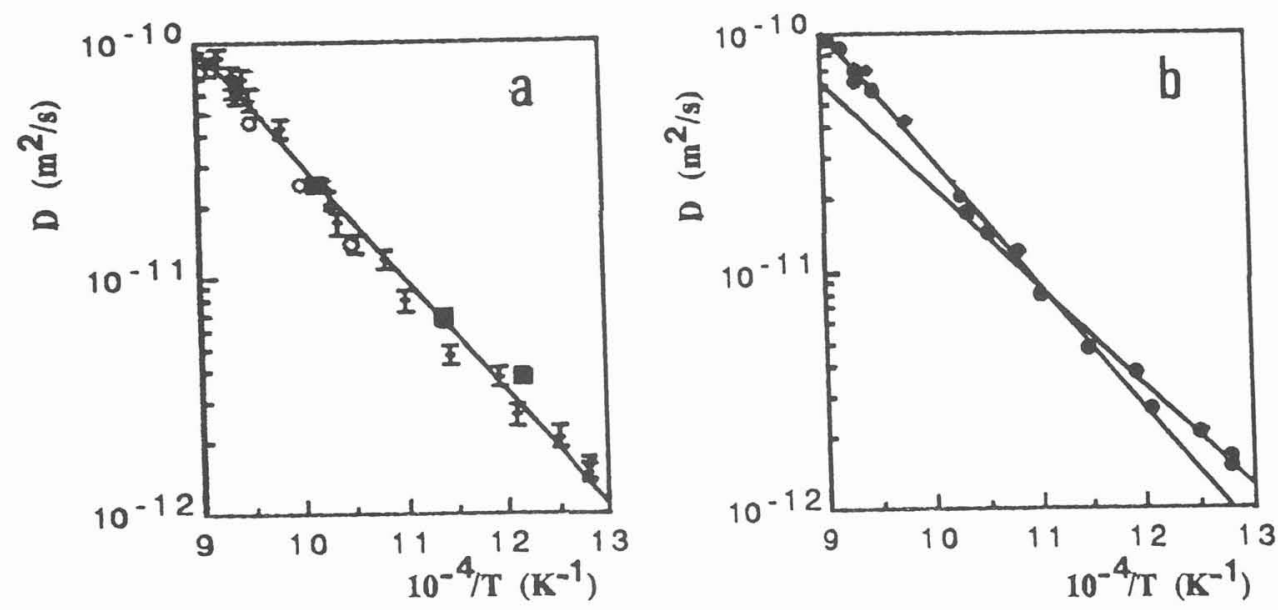

Figure 2: Coefficients de diffusion du zinc dans $\mathrm{CuZn}$ en fonction de linverse de la température absolue (O Inman et al. [3], O Kuper et al. [1], + nos résultats)

Les valeurs des coefficients de diffusion du $65 \mathrm{Zn}$ mesurées par Kuper et al. [1] et par Inman et al. [3] ont, elles aussi, été reportées sur la figure 2 à titre de comparaison.

Pour calculer l'énergie d'activation et le terme de fréquence $D_{0}$, les points expérimentaux ont été traités en utilisant une régression linéaire puisque théoriquement les coefficients de diffusion sont liés à la température par une relation de type Arrhénius. Deux possibilités d'analyse ont été envisagées: 
II- En tenant compte de tous les points expérimentaux (fig. 2a), on obtient la relation :

$$
\mathrm{D}\left(\mathrm{cm}^{2} / \mathrm{s}\right)=0,02 \exp \left(\frac{-92000 \mathrm{~J} / \mathrm{mol}}{\mathrm{RT}}\right)
$$

- En regroupant les neuf résultats relatifs aux huit températures les plus fortes d'une part et le reste des résultats correspondant aux basses températures d'autre part (fig. $2 \mathrm{~b}$ ), on obtient :

$\rightarrow$ à basse température, entre 508 et $692^{\circ} \mathrm{C}$ :

$\mathrm{D}_{1}\left(\mathrm{~cm}^{2} / \mathrm{s}\right)=\mathrm{D}_{1}^{\circ} \exp \left(\frac{-\mathrm{Q} 1}{\mathrm{RT}}\right)=0,0039 \exp \left(\frac{-80700 \mathrm{~J} / \mathrm{mol}}{\mathrm{RT}}\right)$

$\rightarrow$ à haute température, entre 692 et $826^{\circ} \mathrm{C}$ :

$\mathrm{D}_{2}\left(\mathrm{~cm}^{2} / \mathrm{s}\right)=\mathrm{D}_{2}^{0} \exp \left(\frac{-\mathrm{Q} 2}{\mathrm{RT}}\right)=0,13 \exp \left(\frac{-108000 \mathrm{~J} / \mathrm{mol}}{\overline{\mathrm{RT}}}\right)$

où $R$ est la constante des gaz parfaits et $T(K)$ la température de diffusion.

\section{DISCUSSION}

Les coefficients de diffusion mesurés au dessous de la température de transition $\mathrm{A} 2 \Rightarrow \mathrm{B} 2\left(468^{\circ} \mathrm{C}\right)$ sont nettement plus faibles que ceux qui pourraient être extrapolés des hautes températures. Nous ne reviendrons pas sur ce phénomène lié à la présence d'un ordre atomique qui s'établit très rapidement au dessous de $468^{\circ} \mathrm{C}$.

Dans le domaine désordonné $\mathrm{A} 2$ nos résultats pourraient à première vue être uniquement comparés à ceux obtenus par Inman au niveau des paramètres $D_{0}$ et $Q$. Cependant l' analyse de l'ensemble des coefficients de diffusion ( qui conduit à une énergie d'activation moyenne $Q=(92 \pm 3) \mathrm{kJ} / \mathrm{mol}$ avec un coefficient de corrélation $\mathrm{r}^{2}=0,990$ ), montre que la plupart des points expérimentaux sont systématiquement au dessus de la droite de régression aux hautes et basses températures tandis qu'ils se positionnent plutôt au dessous de cette droite aux températures intermédiaires (cf. Fig. 2a). L'analyse au moyen de deux sous domaines (508$692^{\circ} \mathrm{C}$ d'une part et $692-826^{\circ} \mathrm{C}$ d'autre part) permet au contraire i) d'obtenir une meilleure répartition des points expérimentaux sur les deux droites de régression, ii) de rendre compte des deux études antérieures existant dans la littérature où, rappelons le, les travaux avaient été respectivement menés entre $499-718^{\circ} \mathrm{C}$ d'une part [1] et $636-870^{\circ} \mathrm{C}$ d'autre part [3]. Moins de $5 \%$ et $10 \%$ séparent les deux énergies d'activation que nous calculons $\left(\mathrm{Q}_{1}=(80,7 \pm 3) \mathrm{kJ} / \mathrm{mol}\right.$ avec $\mathrm{r}^{2}=0,981 ; \mathrm{Q}_{2}=(108 \pm 3) \mathrm{kJ} / \mathrm{mol}$ avec $\left.\mathrm{r}^{2}=0,986\right)$ en séparant l'ensemble des coefficients de diffusion mesurés dans le domaine A2 en deux sous-domaines de celles respectivement calculées par Kuper d'une part et Inman d'autre part. De plus Kuper mentionne dans son article que les deux coefficients mesurés à haute température sont probablement trop faibles car obtenus sur un alliage présentant une plus faible teneur en zinc.

La présence de deux énergies d'activation suggère que la diffusion est régie par deux mécanismes différents. Les deux défauts impliqués majoritairement dans le transport atomique pourraient être les bilacunes à haute température et les lacunes au dessous de $700^{\circ} \mathrm{C}$. La particularité des présents résultats serait d'impliquer les bilacunes dans un domaine de température plus large que celui où elles interviennent dans les métaux purs. Dans ce cas en effet leur contribution n'a été décelée qu'au voisinage du point de fusion. Une diffusion accélérée par les bilacunes loin du point de fusion a déjà été envisagée pour la diffusion du zinc et du cadmium dans les phases intermétalliques désordonnées $\beta \mathrm{AgCd}$ et $\beta \mathrm{AgZn}$ [4] ainsi que dans les composés intermétalliques totalement ordonnés CoGa et PdIn $[5,6]$.

Dans le cas des composés $\beta \mathrm{AgCd}$ et $\beta \mathrm{AgZn}$, aucune courbure du diagramme d'Arrhénius $\mathrm{Log}(\mathrm{D})=$ $\mathrm{f}(1 / \mathrm{T})$ n'avait pu être mise en évidence dans les domaines désordonnés de structure A2 qui avaient été respectivement étudiés entre $490-650^{\circ} \mathrm{C}$ et $338-563^{\circ} \mathrm{C}$ [4]. L'hypothèse d'une influence des bilacunes sur la diffusion de l'élément le moins noble avait été envisagée pour essayer d'expliquer le fait que dans les deux systèmes les éléments les moins nobles diffusent plus vite que l'argent mais avec une énergie d'activation plus élevée que celle du métal noble. Une énergie d'activation $Q_{A}$ plus faible que $Q_{B}$ liée à des coefficients de diffusion $\mathrm{D}_{\mathrm{A}}$ inférieurs à $\mathrm{D}_{\mathrm{B}}$ est en effet inconcevable dans le cadre du modèle de diffusion lacunaire établi par Manning [7]. 
Par rapport à l'étude précédente relative aux phases $\mathrm{A} 2$ ( $\beta \mathrm{AgCd}$ et $\beta \mathrm{AgZn}$ ), l'hypothèse faisant intervenir des bilacunes dans la migration de l'élément le moins noble est confortée non seulement par l'observation d'une courbure dans le diagramme d'Arrhénius $\log D=f(1 / T)$ mais aussi par le fait que ce défaut est responsable de la dézincification des laitons $\beta(38 \% \mathrm{Zn})$ qui ont la même structure que l'alliage $\mathrm{Cu}-\mathrm{Zn}$ présentement étudié $[8,9,10,11]$. Recemment Qiu et al. [11] ont pu montrer i) que le temps de vie $\tau_{2}$ des positons dans les laitons $\beta$ après dézincification pouvait être attribué au temps de vie en présence de bilacunes, ii) qu'en ralentissant le processus de dézincification par un dopage en Arsenic, l'intensité I 2 du temps de vie $\tau_{2}$ diminuait exactement comme la cinétique de dézincification. Ce résultat est expliqué par les auteurs par le fait qu'en piégeant les bilacunes, la présence de $0,03 \%$ d'arsenic en poids supprime pratiquement la dézincification. Il permet de penser qu'en étudiant la diffusion du ${ }^{65} \mathrm{Zn}$ dans un alliage $\mathrm{Cu}-$ $\mathrm{Zn}(48,0$ at $\% \mathrm{Zn})$ dopé en arsenic, on devrait observer une diminution des coefficients de diffusion du zinc d'un facteur 2 au voisinage de $800^{\circ} \mathrm{C}$. Une étude est en cours pour confirmer cette hypothèse.

En utilisant l'hypothèse d'un mécanisme de diffusion par bilacunes à haute température, (entre 692 et $826^{\circ} \mathrm{C}$ ) on peut estimer l'énergie de migration $\mathrm{H}_{2 \mathrm{~V}}^{\mathrm{m}}$ de la bilacune à partir de l'énergie d'activation $\mathrm{Q}_{2}=$ Q2V. En effet, si $\mathrm{H}_{2 \mathrm{~V}}^{\mathrm{f}}$ est l'enthalpie de formation de la bilacune et si on néglige l'évolution du facteur de corrélation avec la température:

$$
\mathrm{Q}_{2} \approx \mathrm{Q}_{2 \mathrm{~V}} \approx \mathrm{H}_{2 \mathrm{~V}}^{\mathrm{f}}+\mathrm{H}_{2 \mathrm{~V}}^{\mathrm{m}}=2 \mathrm{H}_{1 \mathrm{~V}}^{\mathrm{f}}-\mathrm{H}_{2 \mathrm{~V}}^{\mathrm{b}}+\mathrm{H}_{2 \mathrm{~V}}^{\mathrm{m}}
$$

où $\mathrm{H}_{1 \mathrm{~V}}^{\hat{f}}$ est l'enthalpie de formation de la monolacune et $\mathrm{HkS}(\mathrm{b} ; 2 \mathrm{~V})$ l'énergie de liaison de la bilacune. En prenant $\mathrm{H}_{1 \mathrm{~V}}^{\mathrm{f}}=32,8 \mathrm{~kJ} / \mathrm{mol}$ et $\mathrm{H}_{2 \mathrm{~V}}^{\mathrm{b}}$ égale à $6,7 \mathrm{~kJ} / \mathrm{mol}$ [10], on trouve $\mathrm{H}_{2 \mathrm{~V}}^{\mathrm{m}}=46,7 \mathrm{~kJ} / \mathrm{mol}$.

A basse température, entre 508 et $692^{\circ} \mathrm{C}$, où on suppose l'existence d'un mécanisme de diffusion par monolacunes, $H_{1 \mathrm{~V}}^{\mathrm{m}}$ est déduite de la relation $\mathrm{Q}_{1} \approx \mathrm{Q}_{1 \mathrm{~V}} \approx \mathrm{H}_{1 \mathrm{~V}}^{\mathrm{f}}+\mathrm{H}_{1 \mathrm{~V}}^{\mathrm{m}}$. On trouve ainsi $\mathrm{H}_{1 \mathrm{~V}}^{\mathrm{m}}=49,4 \mathrm{~kJ} / \mathrm{mol}$. Cette valeur est légèrement inférieure à celle donnée par Clark pour $\mathrm{CuZn}$ en phase $\mathrm{B} 2$ trempé à $508^{\circ} \mathrm{C}$ et étudié par résistivité électrique [12]. D'après ces calculs que l'inégalité $\mathrm{H}_{2 \mathrm{~V}}^{\mathrm{m}}<\mathrm{H}_{1 \mathrm{~V}}^{\mathrm{m}}$ semble vérifiée dans cette phase intermétallique comme dans les métaux purs même si la difference est faible.

\section{5 - CONCLUSION}

Dans la phase désordonnée B2 des alliages $\mathrm{CuZn}$ à 48,0 at \% de Zinc, l'évolution des coefficients de diffusion volumiques du zinc peut être représentée par la somme de deux termes exponentiels. A basse température, entre 508 et $692^{\circ} \mathrm{C}$, les défauts responsables de la diffusion semblent être les monolacunes alors que l'accélération de la diffusivité du zinc à haute température, entre 692 et $826^{\circ} \mathrm{C}$, pourraît être expliquée par l'intervention des bilacunes.

\section{REFERENCES}

[1] Kuper A.B., Lazarus D., Manning J.R. et Tomizuka C.T., Phys. Rev. 104 (1956) 1536-1541.

[2] Iijima Y.et Lee C. G., Acta Metall. Mater. 43 (1995) 1183-1188.

[3] Inman M.C., Johnston D., Mercer W.L. et Shuttleworth R., Proceed. Second Radioisotope Conf., Vol. II, (Butterwor Sci. Pub., Londres 1954) pp 85-89.

[4] Benlemlih S., Lexcellent Ch., Moya G. et Bernardini J., Scripta Metall. 24 (1990) 973-978.

[5] Stolwijk N.A., Van Gend M. et Bakker H., Phil. Mag. A 48 (1980) 783-789.

[6] Hahn H., Frohberg G. et Wever H., Phys. Stat. Sol. (a) 79 (1983) 559-566.

[7] Manning J. R.,Phys. Rev. B4 (1971) 1111-1117.

[8] PeeKering H. W., J. Electrochem. Soc. 14 (1967) 698-703.

[9] Revie R. W., Corr. Sci. 12 (1972) 669-671.

[10] Kim S.M. et Buyers W.J.L., Phys. Rev. Lett. 45 (1980) 383-389.

[11] Qiu W. Gan F., Yao L, et Li X., Materials Science Forum 105-110 (1992) 1189-1192.

[12] Clark J.S. et Brown N., J. Phys. Chem. Solids 19. (1961) 291 -296. 